\title{
RELAÇÃO ENTRE A QUALIDADE DE VIDA E O ESTRESSE EM ACADÊMICOS DA ÁREA DA SAÚDE
}

\author{
Rita de Cássia Calderani Borine \\ Instituto de Assistência Médica ao Servidor Público Estadual \\ Kátia da Silva Wanderley \\ Instituto de Assistência Médica ao Servidor Público Estadual \\ Débora Pastore Bassitt \\ Instituto de Psiquiatria do Hospital das Clínicas da FMUSP
}

\begin{abstract}
Resumo
O presente trabalho visou compreender a relação entre a qualidade de vida e o estresse em acadêmicos da área da saúde de uma faculdade privada do interior de Rondônia. Participaram 208 alunos, sendo 133 do sexo feminino e 75 do sexo masculino com idades entre 17 a 55 anos. Para atingir a proposta da pesquisa, foi utilizada a escala Whoqol-Bref para avaliar a qualidade de vida e o Inventário de Sintomas de Stress para Adultos para avaliar o nível de estresse. Conclui-se que os acadêmicos com maior nível de qualidade de vida apresentam menores índices de estresse, sendo os acadêmicos do curso de Medicina Veterinária aqueles com maior índice de qualidade de vida e os acadêmicos do curso de Fisioterapia os que apresentam o maior nível de estresse. Observou-se, também, que o sexo feminino apresentou mais níveis de estresse em comparação ao sexo masculino.
\end{abstract}

Palavras-chave: qualidade de vida, estresse, universitários.

\section{RELATIONSHIP BETWEEN THE QUALITY OF LIFE AND STRESS IN HEALTH AREA STUDENTS}

\begin{abstract}
The present work aimed to comprehend the relationship between quality of life and the stress of healthcare academics from a private college in the interior of Rondônia. Were involved 208 students, with 133 females and 75 males between 17 to 55 years old. To achieve the research proposal the scale used was Whogol-Bref to evaluate the quality of live and the Inventário de Sintomas de Stress para Adultos for the respondent the level of stress. The conclusion is that academics as higher level quality of life lower stress levels, the course of Veterinary Medicine presents highest life quality and the Physiotherapy course is the with highest level of stress, there is also a significant difference between genders, where the female presented more levels of stress comparing to male gender.
\end{abstract}

Keywords: life quality, stress, academics. 


\title{
RELACIÓN ENTRE LA CALIDAD DE VIDA Y EL ESTRÉS EN ACADEMICOS DEL ÁREA DE SALUD
}

\begin{abstract}
Resumen
El objetivo del presente trabajo es de comprender la relación entre la calidad de vida y el estrés de los académicos de la salud na facultad privada del interior de Rondônia. Participaron 208 estudiantes, siendo 133 del sexo femenino y 75 del sexo masculino con edades entre 17 a 55 años. Para alcanzar la propuesta de la investigación fue utilizada la escala Whoqol-Bref para evaluar la calidad de vida y el Inventário de Sintomas de Stress para Adultos para evaluar el nivel de estrés. Se concluye que académicos con mayor calidad de vida presentan menores índices de estrés, siendo el curso de Medicina Veterinaria con mayor calidad de vida y el curso de Fisioterapia con el mayor nivel de estrés, existe también una diferencia significativa entre los sexos, donde el sexo femenino presentó más niveles de estrés en comparación con el sexo masculino.
\end{abstract}

Palabras clave: calidad de vida, estrés, universitarios.

\section{INTRODUÇÃO}

A passagem do Ensino Médio para o Ensino Superior acontece em um período decisivo da evolução do indivíduo, a chamada adolescência, fase está marcada por uma combustão biopsicossocial. $O$ indicador desse processo é a transição do período infanto-juvenil para jovem adulto e as peculiares psicológicas desse desenvolvimento, seus comportamentos e percepções em relação ao meio ambiente e, consequentemente, sua adaptação a ele, período que influencia o processo de desenvolvimento da adolescência no qual está inserido (Cerchiari, 2004).

Segundo Levisky (1995), a transição da adolescência para a vida adulta desenvolve um processo emocional paralelo enquanto se vivencia o luto da perda das características infantis e quando são assumidas as responsabilidades pela própria vida. Esse processo ocorre ao mesmo tempo em que o aluno que frequente uma escola de ensino médio deve fazer uma opção de carreira profissional para prestar os exames vestibulares nas áreas desejadas, antes mesmo de ter sua personalidade formada.

Alguns autores afirmam que os jovens brasileiros vivenciam 0 processo de escolha de profissão por volta dos dezessete aos dezenove anos e ingressam nas universidades ainda em formação da sua personalidade. Consequentemente, eles devem se adaptar ao sistema universitário, o que gera uma série de situações desencadeadoras de processos psicológicos que são manifestados pela evasão escolar, dificuldade de aprendizagem, dependência química, depressão, relacionamentos interpessoais conflituosos e insatisfatórios, vínculos de 
amizades instáveis e prejudiciais, bem como o próprio isolamento e 0 distanciamento familiar (Ferreira, 1986; Klein, 1981; Neiva, 2000).

$O$ processo de inserção na universidade representa um momento em que os indivíduos irão vivenciar novos processos acadêmicos e sociais, a que muitos irão identificar como uma fase difícil e estressante (Baker, 2003). Nesse momento, para passar por essas dificuldades, necessitam de um auxílio para manter essas experiências sociais aceitáveis. O estilo como os acadêmicos lidam com os conhecimentos vivenciados neste momento permanece pautando-a sua saúde física, psicológica e o seu desempenho acadêmico. Assim sendo, o serviço de apoio psicopedagógico tem função de auxiliar e promover repertórios sociais com o intuito de prevenir tais dificuldades.

Os relacionamentos que os acadêmicos constituem em distintos conjuntos representam uma enorme função na ampliação de suas habilidades sociais fundamentais para seu desenvolvimento pessoal e ajustamento psicológico na idade adulta. Enigmas interpessoais estão adjuntos a percepções de enorme desconforto e assíduo ensaio de fuga de circunstâncias sociais, o que compõe fator de risco ao isolamento e à fobia social. Tais estressores e o limitado contato com momentos prazerosos podem contribuir ainda para com o aumento das chances de desenvolvimento de depressão e do ímpeto de abuso de substâncias (Inglés et al., 2005).

Estudantes universitários apresentam, com frequência, problemas psicossociais, tais como: ansiedade, baixa autoestima, depressão, dificuldades de relacionamento, estresse, preocupações excessivas com os estudos gerando um grande índice de evasões que reflete negativamente para o ensino público, evolução do país e para o próprio acadêmico; por conseguinte, tais situações devem ser avaliadas e acompanhadas de forma adequada (Cerchiari, 2004).

Qualidade de vida

Segundo Minayo (1993), o termo qualidade de vida começa a aparecer com relevância na literatura médica após 1975 e, desde então, tem sido muito utilizado, mas sem uma definição precisa. Pode-se apenas inferir, com base nas dimensões ponderadas e reportadas, que abrange "atividades físicas, atividade social e lazer, trabalho, sintomas, renda, cognição, adaptação emocional, autoestima, ansiedade, estresse, atividade sexual, relacionamento interpessoal, impotência, incontinência e satisfação geral com a vida". Não há uma definição clara e definitiva para 
qualidade de vida. Entender-se-á como sendo um conceito abstrato e sujeito a muitas interpretações.

Para Lacaz (2000), foi a partir dessa propagação do termo que o assunto se tornou referência em diversos discursos acadêmicos, políticos e ideológicos movidos pelos interesses governamentais. Existe uma variedade de definições para qualidade de vida as quais, predominantemente, levam em consideração três aspectos, a saber: capacidade funcional, nível socioeconômico e satisfação. No entanto, outros componentes também estão relacionados a ela, tais como estado emocional, atividades intelectuais, capacidade física, interação social, situação econômica e autoproteção de saúde. O termo qualidade de vida é definido como unidimensional; enquanto, para outros autores, é considerado como multidimensional. De qualquer forma, a qualidade de vida é um termo bastante amplo que se relaciona ao bem-estar físico, mental e social.

Segundo Seidl e Zannon (2004) o termo qualidade de vida tem sido cada vez mais empregado quando se avaliam as condições de vida da população com intuito de fazer referência à saúde, a bens materiais da população e ao próprio conforto. Entretanto, vem sendo abordado, também, para delinear situações em que, dentre uma gama de variáveis, buscam-se criar formas em que acrescentam significância e qualidade de vida às pessoas.

São poucos os estudos sobre a temática qualidade de vida junto a universitários no território brasileiro. Segundo Cerchiari (2004) tem-se aplicado considerável importância sobre a qualidade de vida do indivíduo. Porém, apresentar uma qualidade de vida não implica na ausência de doença e sofrimento humano, sendo este um fator que torna a conceitualização da temática muito subjetiva.

\section{Estresse}

Um dos construtos mais relacionados ao sofrimento humano que pode gerar doenças psicossociais dentro de qualquer área social e, mais especificamente, junto aos universitários, é o estresse. Etimologicamente, estresse resulta do latim stringere, significando apertar, cerrar, comprimir. As primeiras referências a esse termo datam do século XIV, cujo significado estava relacionado a sensações de aflição e adversidade. No século XVII, o vocábulo de origem latina passou a ser utilizado em inglês para designar "opressão", "desconforto" e "adversidade" (Rios, 2006). 
Para Chiavenato (1989) o estresse é um conjunto de reações mentais, químicas e físicas de uma determinada pessoa a estímulos ambientais. Sendo assim, o estresse é um conjunto de reações que ocorre em um indivíduo decorrente de estímulos indesejáveis que existem no ambiente. Configura-se como a soma das perturbações orgânicas e psíquicas provocadas por diversos agentes agressores, como trauma, emoções fortes, fadiga, exposição a situações conflitantes.

Já para Miguel e Noronha (2007), o estresse é uma condição imposta pelo próprio indivíduo que se coloca nessa situação por causa de uma oportunidade, restrição ou exigência relacionada ao que ele almeja e cujo resultado pode ser percebido como sendo tanto incerto, quanto importante.

Em relação ao funcionamento do mecanismo de estresse, considerase que existem apenas dois sistemas que conectam todas as partes do organismo, a saber: o sistema nervoso e o sanguíneo. Existe um hormônio em particular, a adrenalina, que é secretado para tornar disponível mais energia para o organismo, acelerar a taxa de pulsação, elevar a pressão cardíaca e, como consequência, elevar também a taxa de circulação de sangue nos músculos; além de estimular o sistema nervoso central. Dessa forma, ao sentir que a situação imediata representa algum tipo de ameaça ou risco, o organismo ativa um estado de alerta, colocando-se em prontidão para emitir uma resposta de luta ou fuga do perigo (Lipp, 1984a, 1994b).

O estresse vem sendo associado a percepções de desconforto. Essas sensações desagradáveis são cada vez mais percebidas pelas pessoas em geral, aumentando o número de indivíduos acometidos pela sintomática (Lipp et al., 1986). Para Lipp et al. (1984), problemas com o sono e cansaço mental também aparecem, podendo ser tanto insônia como sono excessivo; assim como grande é a probabilidade de sono agitado.

$\mathrm{Na}$ área social, pode ocorrer o isolamento, e a consequente falta de amigos e culturalmente pode ocorrer rigidez comprometedora do desempenho em função da grande resistência a mudanças desenvolvida. A criatividade fica prejudicada podendo ocorrer um empobrecimento de valores, principalmente se a pessoa assume uma forte tendência a buscar ou a se manter no poder. A vulnerabilidade ao estresse não se limita apenas ao comprometimento à saúde; pois, mesmo sabendo que o estresse pode gerar diversos problemas relacionados à saúde, é sabido 
que ele gera prejuízo junto à qualidade de vida o que tem, como consequência, a diminuição da produtividade (Abreu et al., 2002).

Segundo Lipp et al. (1984) o estresse pode ser originado de fontes externas e internas. As fontes internas estão relacionadas à maneira de ser do indivíduo, tipo de personalidade e seu modo típico de reagir à vida; uma vez que, muitas vezes, não é o acontecimento em si que se constitui como momento estressante, mas a maneira como é interpretado pela pessoa. Os estressores externos podem estar relacionados às exigências do dia a dia do indivíduo como os problemas de trabalho, familiares, sociais, morte ou doenças de um familiar, perda de uma posição na empresa, não concessão de um objetivo de trabalho, perda de dinheiro ou dificuldades econômicas, notícias ameaçadoras, assaltos e diversas formas de violência nas grandes cidades, entre outros.

Sendo assim, é importante considerar não só a imensa quantidade de fatores potencializadores de estresse, mas também os aspectos individuais, a maneira como cada um reage às pressões cotidianas, bem como os aspectos culturais e sociais aos quais os sujeitos estão submetidos. Fatos como problemas familiares, acidentes, doenças, mortes, conflitos pessoais, dificuldade financeira, desemprego, aposentadoria, problemas no ambiente de trabalho, guerras e inúmeros outros podem ser vivenciados de maneira diversa por dois indivíduos diferentes, em um mesmo contexto histórico, cultural e social, por exemplo; assim como problemas críticos na ordem social de um país podem potencializar o estresse patológico em diversos indivíduos (Lipp, 1994c, 1997d).

O estresse é um dos temas mais discutidos e estudados na atualidade e sua expansão atinge, sem predileções, pessoas de todas as classes, nacionalidades, idades e ocupações. Desse modo, a partir de diversas pesquisas realizadas sobre este tema, evidencia-se que 0 estresse é um dos fatores que mais colaboram com os problemas de saúde. Sendo assim, uma das razões pela qual este tema será abordado neste trabalho (Lipp, 1997).

As fases do estresse

Existem três fases distintas de estresse, sendo a primeira a fase de alerta, a segunda a da resistência e a terceira fase de exaustão as quais se diferenciam por um conjunto de sintomas característicos. Abreu et al. (2002) propuseram o modelo de três fases do estresse, marcado por alerta, resistência e exaustão. 
A primeira fase, Fase de alerta, é analisada como uma fase positiva do estresse. Nessa fase o sujeito se depara, inicialmente, com um agente estressor e uma condição de alerta se coloca fazendo o organismo se arranjar para o que Cannon, em 1939, instituiu de "luta ou fuga" (Lipp, 1997), acontecendo à natural anulação da homeostase. Caso esse estressor tenha uma estabilidade curta, a adrenalina é extinta e acontece o reparo da homeostase, retornando ao estado de origem; assim, seu organismo físico e emocional não terá dificuldade de se adaptar, sem prejuízo ao seu bem-estar. Dessa forma, Lipp (1997) denomina tais adaptações do organismo como estresse sendo um esforço saudável que garante a sobrevivência, o organismo se recupera sem que ocorra agravo maior. É nesta fase que acontece um acréscimo na produtividade e, caso o sujeito não conseguir lidar com a situação, gerar-se-á o estresse de que ele pode usufruir em seu favor em razão da motivação, do entusiasmo e da energia produzidos. Ou seja, é a fase marcada pela forte produção e atuação da adrenalina, fazendo com que o indivíduo fique atento, forte e muito motivado.

A próxima fase, denominada Fase de resistência, ocorre caso a fase anterior prossiga, ou seja, se o estressor é mantido ou se ele é de extensa permanência e ampla veemência; não se constituindo, porém, como maléfico ao organismo. Por meio da ação reparadora o organismo tenta reverter a homeostase. Assim que consegue, os primeiros sintomas desaparecem e o sujeito nota que está melhorando. Se por algum motivo isso não ocorrer, a produtividade sofre uma redução considerável e a vulnerabilidade do dependente aumenta. Nesta segunda fase, o sujeito concentra toda a energia adaptativa para equilibrar-se novamente. Entretanto, se essa reserva é satisfatória, o indivíduo consegue equilibrarse e sair do processo do estresse. Caso o estressor necessite de mais empenho de adaptação, além da competência do indivíduo, o organismo se abranda, ficando suscetível às doenças (Selligman-Silva, 1995).

O sujeito em condição de estresse poderá passar da condição de alerta para o de resistência em questão de segundos. São dois os sintomas que aparecem de maneira muito repentina nesta fase: a sensação de desgaste generalizado sem causa visível e dificuldade com a memória. No nível psicológico, muitas alterações podem acontecer, principalmente, em termos do funcionamento das glândulas suprarrenais: a medula diminui a produção de adrenalina e seu córtex produz corticosteroides. O organismo poderá estar enfraquecido e mais 
vulnerável às doenças; contudo, se o estressor for eliminado, o sujeito poderá voltar ao estado normal, sem sequelas (Lipp \& Guevara, 1994). Muitas doenças começam a aparecer nesta fase e em meio a elas, picos de hipertensão, herpes simples e psoríase e até o diabetes nas pessoas geneticamente predispostas a ele.

A última é a Fase de exaustão, vista por diversos autores desta temática como a mais negativa do estresse. É patológica e acontece quando o estressor persiste por mais tempo ou quando outros estressores ocorrem, ao mesmo tempo, evoluindo o processo. Nesse trajeto, instalase a exaustão psicológica em forma de depressão. A exaustão física desponta e as doenças aumentam com ocorrência, até mesmo, de casos graves. Suas características são aparecimento dos sintomas da primeira fase, além de outros tais como: insônia, problemas dermatológicos, estomacais, cardiovasculares, instabilidade emocional, apatia sexual, ansiedade aguda, inaptidão de tomar decisões, anseio de fugir de tudo, autodúvida e irritabilidade. (Lipp, 1997)

Relação entre qualidade de vida e estresse

Apesar de o estresse e a qualidade de vida constituírem dois conceitos distintos, sua relação tem recebido atenção dos pesquisadores brasileiros, como Abreu et al. (2002) e Lipp et al. (1986). Ela é entendida como um estado de bem-estar físico, mental e social e não só a ausência de doenças. As pessoas que se consideram felizes atribuem sua felicidade ao sucesso em quatro áreas (social, afetiva, saúde e profissional). O estresse, segundo Rios (2006), interfere junto à qualidade de vida, modificando a maneira como o indivíduo interage nas diversas áreas da sua vida; quase sempre ocorrendo na área familiar alta incidência de desajustamentos. O pouco tempo dedicado à família, em função do alto investimento no trabalho, acarreta falta de suporte e apoio quando necessários.

Albuquerque e França (1998) realizaram uma pesquisa, por meio de revisão bibliográfica referente ao estresse e uma pesquisa de campo sobre a qualidade de vida, com o objetivo de investigar a qualidade de vida de funcionários; uma vez que as empresas buscam, cada vez mais, ampliar a produtividade para conseguirem competir com o mercado. Os resultados levaram à conclusão de que a preocupação com a qualidade de vida vem aumentando cada vez mais nas empresas, principalmente junto às empresas que buscam excelência. 
Uma pesquisa realizada com a polícia militar do estado de Minas Gerais teve como objetivo estudar a qualidade de vida no trabalho e estresse ocupacional utilizando, como base, os modelos de Hackman e Oldham (Qualidade de vida no trabalho) e Cooper (Estresse). Neste estudo de caso, com aproximadamente 1.152 colaboradores de um total de 7.607 militares, foram aplicados dois instrumentos para mensurar a qualidade de vida e o estresse a fim de submeter os dados obtidos ao programa estatístico. Chegou-se à conclusão de que existe um elevado nível de estresse decorrente de uma insatisfação com a própria instituição. Em contrapartida, existe um fator que gera qualidade de vida; a satisfação com o trabalho em si. Concluiu-se que existe uma variável determinante de aspectos que contribuem positiva ou negativamente para com a qualidade de vida, sendo possível recomendar à instituição que trabalhasse a cultura organizacional e as políticas de recursos humanos para elevar os níveis de qualidade de vida e, consequentemente, minimizar o estresse (Moraes et al., 2001).

Elias e Navarro (2006) realizaram uma pesquisa com os profissionais da área da saúde, mais precisamente com enfermeiros de um hospital. O objetivo era investigar as relações entre o trabalho, a saúde e as condições de vida daqueles profissionais. Para tanto, realizaram entrevistas semiestruturadas e observações no próprio ambiente de trabalho. Os resultados encontrados apontaram para uma frequência de problemáticas relacionadas à saúde física e emocional decorrentes do estresse.

A pesquisa de Alves et al. (2010) teve como objetivo comparar a qualidade de vida dos acadêmicos do primeiro e último período do curso de Medicina de uma universidade na cidade de Recife. Por meio de aplicação de um instrumento criado para mensurar a qualidade de vida, Whoqol-bref, com aproximadamente 370 estudantes, sendo que $62 \%$ da população participante estavam matriculadas no primeiro período e $38 \%$ no último período. Os resultados permitiram concluir que, durante a graduação, ocorre um desgaste no quesito emocional, trazido pelo teste, como domínio psicológico. Os demais domínios (físico, ambientes e sociais) não apresentaram diferenças significativas. 


\section{MÉTODO}

\section{Participantes}

Participaram da pesquisa 208 universitários, sendo 75 do sexo masculino e 133 do sexo feminino dos cursos de saúde, a saber: Educação Física, Enfermagem, Farmácia, Fisioterapia, Medicina, Medicina Veterinária e Odontologia de uma Faculdade privada de Rondônia, de ambos os sexos, sem nenhuma restrição de idade.

\section{Instrumentos}

Para alcançar os objetivos estabelecidos inicialmente, foi utilizado o Termo de Consentimento Livre Esclarecido definido pela Resolução n. 196/96 do Conselho Nacional de Saúde e a Resolução do CFP n.016/2000 de 20 de dezembro de 2000.

O instrumento escolhido para avaliar a qualidade de vida é o Whoqol - bref (Wordl Health Organization Quality of Life Instrument Bref) indicado para adultos. Foi utilizada a forma abreviada que contém 26 questões, das quais duas estão relacionadas a aspectos gerais. As outras 24 questões representam os domínios que compõem o instrumento original, a saber: Meio-ambiente, Relações sociais, Domínio Psicológico e Domínio Físico.

De acordo com Orlandino (2008), o Inventário de Sintomas de Stress para Adultos -ISSL é um instrumento que busca identificar a sintomatologia do paciente; se este possui sintomas de estresse, o tipo de sintoma e a fase em que se situa: (1) alerta, (2) resistência, (3) exaustão. $\mathrm{O}$ instrumento baseou-se nos sintomas característicos - físicos e psicológicos - de cada fase do estresse.

\section{Procedimentos}

A presente pesquisa foi exposta para avaliação do Comitê de Ética em Pesquisa de uma instituição privada de Rondônia (número de protocolo é 977-12). Após a aprovação, iniciou-se a aplicação dos questionários com os acadêmicos matriculados nos cursos da área da saúde. Depois de sanadas todas as dúvidas dos participantes, foram assinadas duas vias do Termo de Consentimento Livre e Esclarecido e aplicados os instrumentos Inventário Sintomas de Stress para Adultos ISSL e Whoqol-Bref. 


\section{RESULTADOS}

A Tabela 1 apresenta a estatística descritiva das médias dos cursos com relação à qualidade de vida, estresse e suas respectivas fases. Conforme se observa na Tabela, o curso de Medicina Veterinária apresentou o melhor índice de qualidade de vida e o de Educação Física, o pior índice. Os cursos de Enfermagem, Farmácia, Medicina, Odontologia e Fisioterapia apresentaram índices muito próximos. Conforme previsto, o curso de Medicina Veterinária apresentou o menor índice de estresse, já que, conforme visto, apresentou uma qualidade de vida maior que os demais. Entretanto, o curso de Educação Física aparece em segundo lugar em termos de estresse, apesar de ser o curso com menor qualidade de vida.

Tabela 1. Análise estatísticas descritivas das médias de desempenho dos cursos em termos de qualidade de vida e estresse

Cursos

$\mathrm{N}$ Qualidade Estresse Fase de vida alarme

\begin{tabular}{ccccccc}
\hline $\begin{array}{c}\text { Educação } \\
\text { Física }\end{array}$ & 29 & 88,6 & 9,44 & 2,58 & 3,96 & 2,90 \\
\hline Enfermagem & 34 & 91,3 & 12,64 & 3,43 & 4,65 & 4,56 \\
\hline Farmácia & 35 & 91,6 & 9,97 & 2,59 & 4,05 & 3,31 \\
\hline Medicina & 23 & 91,8 & 11,30 & 2,78 & 4,22 & 4,30 \\
\hline Odontologia & 31 & 92,0 & 13,67 & 4,16 & 5,06 & 4,45 \\
\hline Fisioterapia & 26 & 93,1 & 15,11 & 4,31 & 5,34 & 5,46 \\
\hline $\begin{array}{c}\text { Medicina } \\
\text { Veterinária }\end{array}$ & 30 & 97,3 & 9,27 & 2,72 & 3,70 & 2,83 \\
\hline
\end{tabular}

Foi realizado para verificação das diferenças de média do estresse e qualidade de vida para os cursos utilizando o método estatístico de Tukey, que testa a existência de homogeneidade ou não entre as médias dos cursos. Por meio desta análise, não houve diferenciação para qualidade de vida e ficou constatada uma divisão em dois grupos diante das médias de estresse dos cursos, sendo um com menores médias de estresse (Grupo 1) e o outro com maiores médias de estresse (Grupo 2). Os cursos com menor índice de estresse são os cursos de Medicina Veterinária e Educação Física, seguidos pelos cursos de Farmácia, Medicina, Enfermagem e Odontologia. O curso com maior índice de estresse apresentado é de Fisioterapia, conforme se verifica na Tabela 2: 
Tabela 2. Teste Tukey - Diferenças de média dos cursos em relação ao estresse

\begin{tabular}{|c|c|c|c|}
\hline \multirow[t]{2}{*}{ Cursos } & \multirow[t]{2}{*}{$\mathrm{N}$} & \multicolumn{2}{|c|}{$\begin{array}{c}\text { Subconjunto para alfa }= \\
0.05\end{array}$} \\
\hline & & $\begin{array}{c}\text { Média Grupo } \\
1\end{array}$ & $\begin{array}{l}\text { Média Grupo } \\
2\end{array}$ \\
\hline Medicina Veterinária & 30 & 9,27 & \\
\hline Educação Física & 29 & 9,44 & \\
\hline Farmácia & 35 & 9,97 & \\
\hline Medicina & 23 & & 11,30 \\
\hline Enfermagem & 34 & & 12,64 \\
\hline Odontologia & 31 & & 13,67 \\
\hline Fisioterapia & 26 & & 15,11 \\
\hline
\end{tabular}

Foi utilizado o método estatístico teste-t para amostras independentes para verificar a existência de diferenças de médias de qualidade de vida e estresse por sexo. Percebeu-se que as mulheres têm menor qualidade de vida comparada aos homens e também maior nível de estresse. A Tabela 3 resume os resultados.

Tabela 3. Teste-t - Diferenças de média de Qualidade de Vida e Estresse por sexo

\begin{tabular}{|c|c|c|c|c|c|c|}
\hline & Sexo & $\mathrm{N}$ & Média & $\begin{array}{l}\text { Desvio } \\
\text { Padrão }\end{array}$ & $\mathrm{T}$ & $p$ \\
\hline \multirow{2}{*}{$\begin{array}{l}\text { Qualidade de } \\
\text { Vida }\end{array}$} & Masculino & 75 & 96,3 & 10,67 & \multirow[b]{2}{*}{3,875} & \multirow[b]{2}{*}{0,00} \\
\hline & Feminino & 133 & 89,9 & 11,94 & & \\
\hline \multirow[t]{2}{*}{ Estresse } & Masculino & 75 & 7,9 & 5,48 & \multirow[b]{2}{*}{$-5,697$} & \multirow[b]{2}{*}{0,00} \\
\hline & Feminino & 133 & 13,6 & 7,54 & & \\
\hline
\end{tabular}

Para verificar a correlação entre qualidade de vida e o estresse, foi realizada uma correlação de Pearson entre o Inventário de Sintomas de Estresse para Adultos - ISSL e Whoqol-Bref, tanto para verificação dos resultados de forma global entre estresse e qualidade de vida, como também entre qualidade de vida e cada fase do estresse. Uma correlação moderada negativa moderada foi encontrada entre qualidade de vida e estresse dos acadêmicos da área da saúde, implicando que quanto maior o escore em qualidade de vida, menor os indícios de estresse entre acadêmicos $(r=-0,478 ; p<0,01)$. 
Quando correlacionada a qualidade de vida com as três fases do estresse verificado no instrumento ISSL, ou seja, fase de alerta, fase de resistência e fase de exaustão, a maior correlação da qualidade de vida foi com a fase de resistência $(r=-0,491 ; p<0,05)$, seguida pela fase de exaustão $(r=-0,444 p<0,05)$ e com a fase de alerta $(r=-302 ; p<0,05)$, que corresponde à fase positiva do estresse.

\section{DISCUSSÃO}

Esta pesquisa evidenciou a relação entre o nível de estresse e qualidade de vida entre os acadêmicos da área de saúde. Constatou-se que os três fatores referentes ao estresse, alerta, resistência e exaustão, apresentaram uma correlação negativa com a qualidade de vida. Isso demonstra que, quanto maior a qualidade de vida, menor foi o nível de estresse pelos acadêmicos. Os resultados estão de acordo com as pesquisas de diversos autores (Lipp \& Tanganelli, 2002; Sadir et al., 2010; Loureiro et al., 2008).

Quando se comparam os escores médios dos acadêmicos quanto à vulnerabilidade ao estresse e qualidade de vida entre os cursos da área de saúde, o curso que apresentou maior qualidade de vida foi o de Medicina Veterinária, enquanto que o com menor índice de qualidade de vida foi Educação Física. Tais resultados corroboram com a pesquisa realizada por Pfuetzenreiter e Zylbersztajn (2008) com estudantes do curso de Medicina Veterinária, que objetivou compreender a percepção do estudante sobre a atuação do médico veterinário na área da saúde. A significativa importância que os próprios acadêmicos de Medicina Veterinária atribuíram à sua profissão foi tratada como um fator motivacional e que tende a aumentar sua qualidade de vida. Como menciona Lacaz (2000) o fator motivação é essencial para proporcionar bem-estar ao indivíduo, consequentemente, aumento da qualidade de vida.

O curso de Educação Física apresentou a menor média em qualidade de vida. O estudo de Chiapetti e Serbena (2007) objetivou investigar o uso de álcool, tabaco e drogas por alunos da área de saúde. Nesse estudo, um dos cursos pesquisados foi o curso de Educação Física, que apresentou um elevado uso de substâncias lícitas e ilícitas para cuja utilização, inicialmente, foi atribuída à busca de "prazer e diversão". Como segundo motivo mais comum, observou-se a utilização para fins de melhoria de desempenho, apontando o uso de anabolizante para alcançar as metas e padrões estéticos, o que influenciava, negativamente, a qualidade de vida 
dos acadêmicos. Na presente pesquisa realizada não tivemos controle das variáveis que podem influenciar a qualidade de vida dos acadêmicos de educação física.

Em relação ao estresse, o curso que apresentou maior nível foi o curso de Fisioterapia e o curso com menor nível foi o curso de Medicina Veterinária. Uma pesquisa realizada com os acadêmicos do quarto período do curso de Fisioterapia de uma universidade estadual que teve como objetivo verificar a prevalência da síndrome de burnout. Apresentou em seus resultados escores moderados referentes à exaustão emocional e despersonalização por parte dos acadêmicos de Fisioterapia (Christofoletti et al., 2007). Aparentemente, o curso pode apresentar variáveis específicas e ainda não determinadas que possam promover aos seus acadêmicos situações de desconforto emocionais como estresse, exaustão emocional e despersonalização.

Quando observada a diferença de estresse e qualidade de vida por sexo, os homens apresentaram maior qualidade de vida e um menor nível de estresse. Na pesquisa de Calais, Andrade e Lipp (2003), cujo objetivo era o de verificar a prevalência do estresse pela escolaridade e sexo em jovens adultos, obteve-se, como resultado, que o sexo feminino tem maior índice de estresse quando comparado ao sexo masculino, resultados este corroborado pelo fato de as mulheres demonstrarem sintomas expressivos de estresse. Como menciona Areias e Guimarães (2004), as mulheres ficam sujeitas a uma dupla sobrecarga de trabalho, ocorrendo multiplicidade de papéis (casamento, filhos e demandas ocupacionais) o que poderia contribuir com níveis significativos de estresse. Finalmente, é importante ressaltar que a combinação entre o trabalho excessivo, uma situação econômica menos favorecida e uma dificuldade nos repertórios de resposta para enfrentamento de situações de desgaste emocional faz com que as mulheres apresentem maior nível de estresse que os homens e, consequentemente, uma qualidade de vida inferior ao sexo oposto (Levi, 1999).

\section{CONSIDERAÇÕES FINAIS}

A vida acadêmica de alunos em busca de formação profissional é extremamente agitada. Diariamente, esses indivíduos recebem uma carga de informações novas que os obriga a viver, a todos os momentos, em busca da atualização. Constantemente, encontramo-nos frente a transformações que exigem adaptações o que, invariavelmente, gera 
desgaste físico e emocional. Alguns acadêmicos conseguem se adaptar facilmente à nova rotina de estudos e trabalho; porém essas constantes mudanças e adaptações são fatores que influenciam negativamente sua qualidade de vida, podendo propiciar, com maior frequência, o surgimento do estresse.

Conclui-se que existe uma correlação moderada entre a qualidade de vida e o estresse dos alunos universitários dos cursos da saúde, constatação esta que constituía o principal objetivo da pesquisa. Outro dado importante apontado na pesquisa foi o fato de os homens terem melhor qualidade de vida em comparação às mulheres que, consequentemente, apresentam dados elevados de estresse. Esse fator pode estar relacionado ao acúmulo de funções que o sexo feminino exerce; como cuidar da casa, realizar um trabalho assalariado, estudar, conquistar seu espaço no mercado de trabalho, entre outras situações.

$O$ processo de adaptação ao ensino superior apresenta uma baixa qualidade de vida segundo Saupe et al. (2004); uma vez que, nesse momento, os acadêmicos estão passando por diversas situações novas, tais como o processo de adaptação aos estudos, ao mecanismo de ensinoaprendizagem da instituição, a dupla ou tripla jornada, bem como as formas de avaliação, a própria interação com os colegas de sala a que podemos chamar de habilidade social e aceitação das diferenças.

Apesar de existir um número significativo de pesquisas sobre os construtos qualidade de vida e estresse separadamente; são poucos estudos sobre a correlação dos construtos com o público universitário. Com pesquisas mais aprofundadas sobre a temática, poderíamos propor, com embasamento científico, alterações nos cursos de graduação; desde o formato do curso, ensino-aprendizagem e, até mesmo com relação aos incentivos para os estudos. Desse modo, poder-se-ia contribuir para com um melhor aproveitamento do curso, bem como proporcionar à sociedade, indivíduos mais bem qualificados e capacitados para exercerem suas profissões. Afinal, não é apenas a sociedade que perde com um profissional mal qualificado, o que já seria uma situação grave; o próprio indivíduo, ou seja, o universitário, em razão da baixa qualidade de vida e eventos estressores contínuos, poderá sair para o mercado de trabalho adoecido. 


\section{REFERÊNCIAS}

Abreu, K. L. [et al.] (2002). Estresse ocupacional e Síndrome de Burnout no exercício profissional da psicologia. Psicologia Ciência e Profissão, 22(2), 22-29. doi: 10.1590/S1414-98932002000200004

Albuquerque, L. G., \& França, A. C. L. (1998). Estratégias de recursos humanos e gestão de qualidade de vida no trabalho: o stress e a expansão do conceito de qualidade total. Revista de Administração da Universidade de São Paulo, 33(2), 40-51.

Alves, J. G. B., Tenório, M., Anjos, A. G., \& Figueroa, J. N. (2010). Qualidade de vida em estudantes de Medicina no início e final do curso: avaliação pelo Whoqol-bref. Revista brasileira de educação médica, 34 (1), 91-96. doi: 10.1590/S0100-55022010000100011

Areias, M. E. Q., \& Guimarães, L. A. M. (2004). Gênero e estresse em trabalhadores de uma Universidade Pública do Estado de São Paulo. Psicologia em Estudo, Maringá, 9(2), 255-262. doi: 10.1590/S141373722004000200011

Baker, S. R. (2003). A prospective longitudinal investigation of social problemsolving appraisals on adjustment to university, stress, health and academic motivation and performance. Personality and Individual Differences, 35, 569-591. doi: 10.1016/S0191-8869(02)00220-9

Calais, S. L., Andrade, L. M. B., \& Lipp, M. E. N. (2003). Diferenças de Sexo e Escolaridade na Manifestação de Stress em Adultos Jovens. Psicologia: Reflexão e Crítica, 16(2), 257-263. doi: 10.1590/S010279722003000200005

Cerchiari, E. A. N. (2004). Saúde mental e qualidade de vida em estudantes universitários. (Tese de doutorado / UNICAMP - Universidade Estadual de Campinas).

Recuperado

de

http://www.bibliotecadigital.unicamp.br/document/?code=vtls000341653\&f $\mathrm{d}=\mathrm{y}$

Chiapetti, N., \& Serbena, C. A. (2007). Uso de Álcool, Tabaco e Drogas por estudantes da Área de Saúde de uma Universidade de Curitiba. Psicologia: Reflexão e Crítica, 20(2), 303-313. doi: 10.1590/S010279722007000200017

Chiavenato, I. (1989). Recursos Humanos na empresa (1. Ed.). São Paulo: Atlas.

Christofoletti, G., Trelha, C. S., Galera, R. M., \& Feracin, M. A. (2007). Síndrome de burnout em acadêmicos de fisioterapia. Fisioterapia e pesquisa, 14(2), 35-39. doi: 10.1590/fpusp.v14i2.75857 
Elias, M. A., \& Navarro V. L. (2006). A relação entre o trabalho, a saúde e as condições de vida: negatividade e positividade no trabalho das profissionais de enfermagem de um hospital escola. Revista Latino-americana de Enfermagem, 14(4), 517-525. doi: 10.1590/S0104-11692006000400008

Ferreira, A. B. H. (1986). Novo dicionário da Língua Portuguesa. Rio de Janeiro: Nova Fronteira.

Fleck, M. P. A., Louzada, S., Xavier, M., Chachamovich, E., Vieira, G., Santos, L., \& Pinzon, V. (2000). Aplicação da versão em português do instrumento abreviado de avaliação da qualidade de vida "WHOQOL-bref". Revista Saúde Pública, 34(2), 178-183. doi: 10.1590/S0034-89102000000200012

Inglés, C. J., Hidalgo, M. D., \& Méndez, F. X. (2005). Interpersonal difficulties in adolescence: A new self-report measure. Europe na Journal of Psychologycal Assessment, 21(1), 11-22. doi: 10.1027//1015-5759.21.1.11 Klein, M. (1981). Psicanálise da criança. São Paulo: Mestre Jou.

Lacaz, F. A. C. (2000). Qualidade de vida no trabalho e saúde/doença. Ciência e Saúde Coletiva, 5(1), 151-161. doi: 10.1590/S1413-81232000000100013

Levi, L. (1999). Guía sobre el estrés relacionado con el trabajo: La "sal de la vida" o el "beso de la muerte?". Barcelona: Dirección General de Empleo y Asuntos Sociales de la Comisión Europea de Seguridad y Salud en el Trabajo.

Levisky, D. L. (1995). Adolescência - Reflexões psicanalíticas. Porto Alegre: Artes Médicas.

Lipp, M.E. N. (1984). Stress e suas implicações. Estudos de Psicologia, (3), 5-19.

Lipp, M. E. N., Romano, A. S. P. F., Covolan, M. A. \& Nery, M. I. (1986). Como enfrentar o stress. São Paulo: Ícone.

Lipp, M. E. N., \& Guevara, A. J. H. (1994). Validação empírica do Inventário de Sintomas de Stress. Estudos de Psicologia, 11(3), 42-49.

Lipp, M. E. N. (1997). Pesquisas sobre stress no Brasil. Campinas: Papirus.

Lipp, M. E. N., \& Tanganelli, M. S. (2002). Stress e Qualidade de vida em Magistrados da Justiça do trabalho: Diferenças entre homens e mulheres. Psicologia: Reflexão e Crítica, 15(3), 537-548. doi: 10.1590/S010279722002000300008

Loureiro, E., Mcintyre, T. Mota-Cardoso, R. \& Ferreira, M. A. (2008). A relação entre o stress e os estilos de vida: nos estudantes de medicina da faculdade de medicina do Porto. Acta Med Port, 21, 209-214.

Miguel, F. K., \& Noronha, A. P. P. (2007). Estudo dos Parâmetros Psicométricos da Escala de Vulnerabilidade ao Estresse no Trabalho. Revista Evaluar, (7), 1-18.

Minayo, M. C. de S. (1993). O desafio do Conhecimento. São Paulo: Hucitec. 
Moraes, L. F. R., Pereira, L. Z., Lopes, H. E. G., Rocha, D. B., Ferreira, S. A. A., \& Portes, P. C. P. (2001). Estresse e qualidade de vida no trabalho da polícia militar do Estado de Minas Gerais. Recuperado de http://www.anpad.org.br/admin/pdf/enanpad2001-grt-359.pdf

Neiva, K. M. C. (2000). Escala de maturidade para a escolha profissional (EMEP) PSIC- São Paulo, 1(3), 28-33.

Organização Mundial da Saúde. (2003) Classificação internacional de funcionalidade, incapacidade e saúde. São Paulo: Edusp.

Orlandino, A. (2008). O stress ocupacional em professores do Ensino Médio (Dissertação de Mestrado em Educação - Universidade do Oeste Paulista UNOESTE) Presidente Prudente/SP. Recuperado de http://apeclx.unoeste.br/tede/tde_busca/arquivo.php?codArquivo $=123$

Pfuetzenreiter, M. R., \& Zylbersztajn, A. (2008). Percepções de estudantes de medicina veterinária sobre a atuação na área da saúde: um estudo baseado na idéia de "estilo de pensamento" de Ludwik Fleck. Ciência \& Saúde Coletiva, 13(2), 2105-2114.

Rios, O. F. L. (2006). Níveis de estresse e depressão em estudantes universitários. Dissertação de mestrado. Pontifícia Universidade Católica, São Paulo.

Sadir, M. A., Bignotto, M. M., Lipp, M. E. N. (2010). Stress e qualidade de vida: influência de algumas variáveis pessoais. Paideia, 20(45), 73-81. doi: 10.1590/S0103-863X2010000100010

Saupe, R., Nietche, E. A., Cestari, M. E., Giorgi, M. D. M., Krahl, M. (2004). Qualidade de vida dos acadêmicos de enfermagem. Revista Latinoamericana de Enfermagem, 12(4), 636-342. doi: 10.1590/S010411692004000400009

Seidl, E. M. F., \& Zannon, C. M. L. da C. (2004). Qualidade de vida e saúde: aspectos conceituais e metodológicos. Caderno da Saúde Pública, 20(2), 580-588. doi: 10.1590/S0102-311X2004000200027

Selligman-Silva, E. (1995). Psicopatologia e psicodinâmica no trabalho. In: Mendes R. Patologia do trabalho. Rio de Janeiro: Atheneu. 287-310.

Whoqol group (1994). The development of the World Health Organization quality of life assessment instrument (the WHOQOL). In: Orley J., Kuyken W., (editors). Quality of life assessment: international perspectives. Heigelberg: Springer Verlag. p 41-60. 
Sobre os autores

Rita de Cássia Calderani Borine é psicóloga, mestranda pelo Instituto de Assistência Médica ao Servidor Público Estadual. rcalderani@bol.com.br

Kátia da Silva Wanderley é psicóloga do Instituto de Assistência Médica ao Servidor Público Estadual. katizpsi@uol.com.br

Débora Pastore Bassitt é médica do Instituto de Psiquiatria do Hospital das Clínicas da FMUSP. dbassitt73@gmail.com

Recebido em: 08/05/2015

Revisado em: 04/08/2015

Aceito em: $14 / 08 / 2015$ 